\title{
Laser photomodification of insulin solution
}

\author{
Anna V. Neupokoeva ${ }^{1 *}$, Evgenia A. Kuzina ${ }^{1}$, Nikita S. Moskalev ${ }^{1}$, Anna I. Nikonova ${ }^{1}$, \\ and Sergey A. Nebogin² \\ ${ }^{1}$ Department of Medical and Biological Physics, Irkutsk State Medical University, 1 Krasnogo Vosstaniya St, \\ Irkutsk 664009, Russia \\ ${ }^{2}$ Department of Quantum Physics and Nanotechnology, Irkutsk National Research Technical University, \\ 83 Lermontov St., Irkutsk 664074, Russia
}

* e-mail: annett_2005@inbox.ru

\begin{abstract}
The article discusses influence of laser radiation on structure of insulin solution. We monitored changes in the structure of insulin solutions by means of analysis of crystallograms with a nanometer resolution probe microscope as well as by correlation processing of speckle patterns obtained by passing laser radiation through the solution. It was experimentally shown that laser radiation promoted solution homogenization by changing size of macromolecular clusters. (C) 2019 Journal of Biomedical Photonics \& Engineering.
\end{abstract}

Keywords: crystallogram; image processing; structure monitoring; laser biostimulation.

Paper \#3314 received 10 Feb 2019; revised manuscript received 18 Mar 2019; accepted for publication 15 Apr 2019; published online 13 May 2019. doi: 10.18287/JBPE19.05.020301.

\section{Introduction}

Laser radiation has been used in medicine for several decades and currently has a number of methods differing time and location of exposure, wavelength and radiation power [1]. At the same time, there is no generally accepted theory explaining mechanisms of laser therapy. But ways of detecting changes that occur under action of laser radiation recently have significantly expanded. Thus, different experimental techniques of structural changes of complex organic solutions such as Raman spectroscopy and IR spectroscopy, crystallographic method and method of speckle patterns processing have become widespread in practice [2-8]. Using new research methods has led to appearance of some new theories about the mechanisms of action of laser radiation on living organisms [1, 4, 9, 10]. In particular, in Ref. [4] primary action of laser radiation was considered as a result of interaction with blood proteins. In Ref. [10] the authors supposed that laser radiation reduced macromolecular clusters that formed protein molecules in water solution and thus changed their functional activity. In addition, in article [10] it was shown that laser radiation could affect molecular clusters not only in biological fluids, but also in drugs composition that leads to increasing their effectiveness.

Therefore, the purpose of this work is to study effect of laser radiation on the microstructure of an insulin solution. The choice of the object of research was due to two main reasons. First, it is nature of an insulin molecule formed by two polypeptide chains, which contains 51 amino acid residues. Six insulin molecules are bound in a hexamer. Molecules are hold together by histidine residues bound by zinc ions. Injected insulin is under the skin in the form of a hexamer, which gradually breaks down into biologically active monomers that enter the bloodstream. Secondly, according to the World Health Organization, the number of diabetics is more than $3 \%$ of the world's population. Moreover, a significant percentage of these patients are forced to take daily drugs (in particular, insulin) to decreasing their blood glucose levels. Therefore, it is important to develop methods aimed at improving insulin effectiveness in the body and reducing insulin consumption.

\section{Materials and methods}

The method of crystallogram analysis was used for adequate assessment of structural changes occurring under action of laser radiation in biosimilar liquids as one of the simplest methods of structural changes diagnostics. Crystallograms of biological fluids were formed due to dehydration self-organization of protein solutions and other substances $[6,11]$ during drying of solution droplets on a solid substrate. It was found that the resulting patterns correlate well with the signs of 

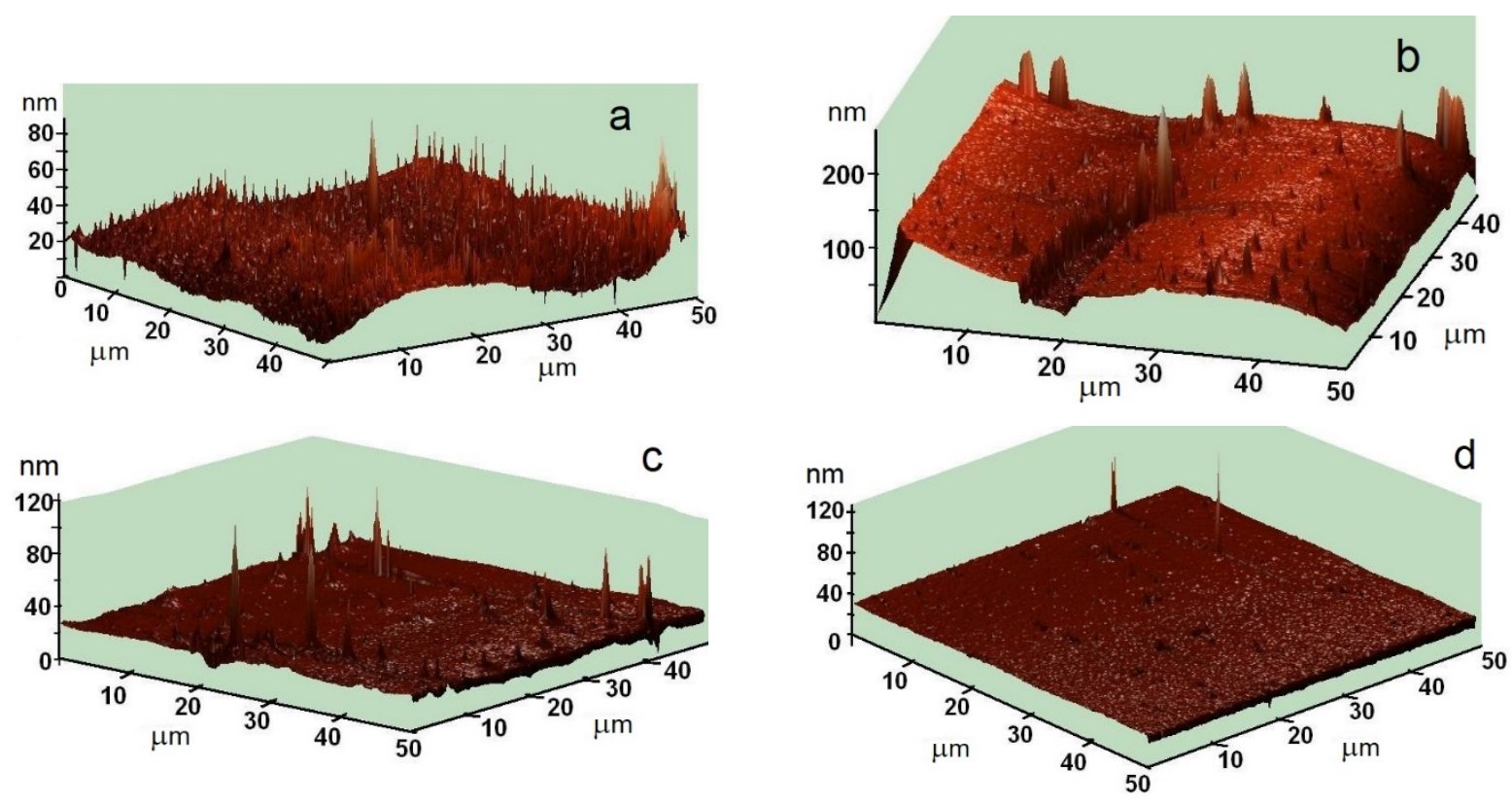

Fig. $13 \mathrm{D}$ model of the insulin solution crystallogram surface: a - control (before exposure), b - after 5 minutes, $\mathrm{c}-$ after 10 minutes, $\mathrm{d}$ - after 15 minutes.

patients' diseases $[6,12,13]$. Therefore, the method of crystallogram analysis can be used to record changes occurring in biological fluids under laser radiation exposure. The detecting capability of laser-induced changes in serum and plasma was shown in Ref. [5]. A possible mechanism of laser-induced changes is decreasing bioorganic molecules (primarily macromolecules) clusters size under the influence of laser radiation [5].

Usually crystallogram analysis is carried out by an optical microscope. In this case the geometric dimensions (longitudinal and transverse) of the characteristic structures of the crystallogram are estimated, while information about the relief (surface profile) is usually lost. That is why, in this paper the analysis of crystallograms was carried out by a probe microscope to obtain information about threedimensional structure of the crystallogram.

As an alternative method of changes recording in the microstructure of the protein solution, the "chessboard" method for speckle patterns processing was chosen. Since protein clusters in the solution form optical inhomogeneities, when laser radiation passes through a cell with such solution, a speckle pattern is formed, and a characteristic speckle size depends inversely on the inhomogeneity (cluster). To determine the characteristic speckle size, the correlation coefficient of a twodimensional speckle image and a regular structure ("chessboard") was calculated. Paper [14] presents a program that calculates the correlation coefficient of a speckle image and "chessboards" with various sizes from $5 \times 5$ to $100 \times 100$ pixels in 5 pixel increments until the most clearly expressed structure is obtained. The chessboard, with the most clear structure and the biggest correlation coefficient was taken as a standard. Then every speckle image was analysed with this standard: the correlation coefficient of the reference image and each of the analyzed images was calculated.

The research object was a solution of human insulin in the form of the drug "Actrapid" in ampoules. The insulin solution was poured into a cuvette and exposed by laser irradiation with a wavelength of $655 \mathrm{~nm}$ and intensity $150 \mathrm{~mW} / \mathrm{cm}^{2}$. The exposure time was $2,5,10$, 15 minutes. Before irradiation, a control (non-exposed) sample was poured onto the glass plate. Then, after each irradiation time, several drops of the solution were also poured onto a glass substrate. All samples were kept at room temperature to obtain crystallograms. Crystallogram of the insulin solution is a thin transparent film with inhomogeneities invisible not only by naked eye, but also by an optical microscope. Therefore, the obtained crystallograms were investigated in a scanning probe microscope NT-MDT solver (contact scanning method).

\section{Results}

3D models of crystallogram relief (Fig. 1) were built using a probe microscope. The increasing time of laser exposure caused significant decreasing the number of crystallogram peaks and average peak height and increased the layer smoothness. So, before exposure, the average height of the peak was $50-60 \mathrm{~nm}$, and the maximum - about $80 \mathrm{~nm}$. After 5- and 10-minute exposure, the peak height did not change significantly, but their frequency went down significantly: from 60-80 to $100 \mu \mathrm{m}^{2}$ in the control, to $5-8$ per $100 \mu \mathrm{m}^{2}$ after 10 minutes of radiation. After 15-minute exposure the average peak height became $30 \mathrm{~nm}$ and peak frequency reduced to 3-4 per $100 \mu \mathrm{m}^{2}$.

A significant increasing the smoothness of crystallogram layer was caused by the conformational 

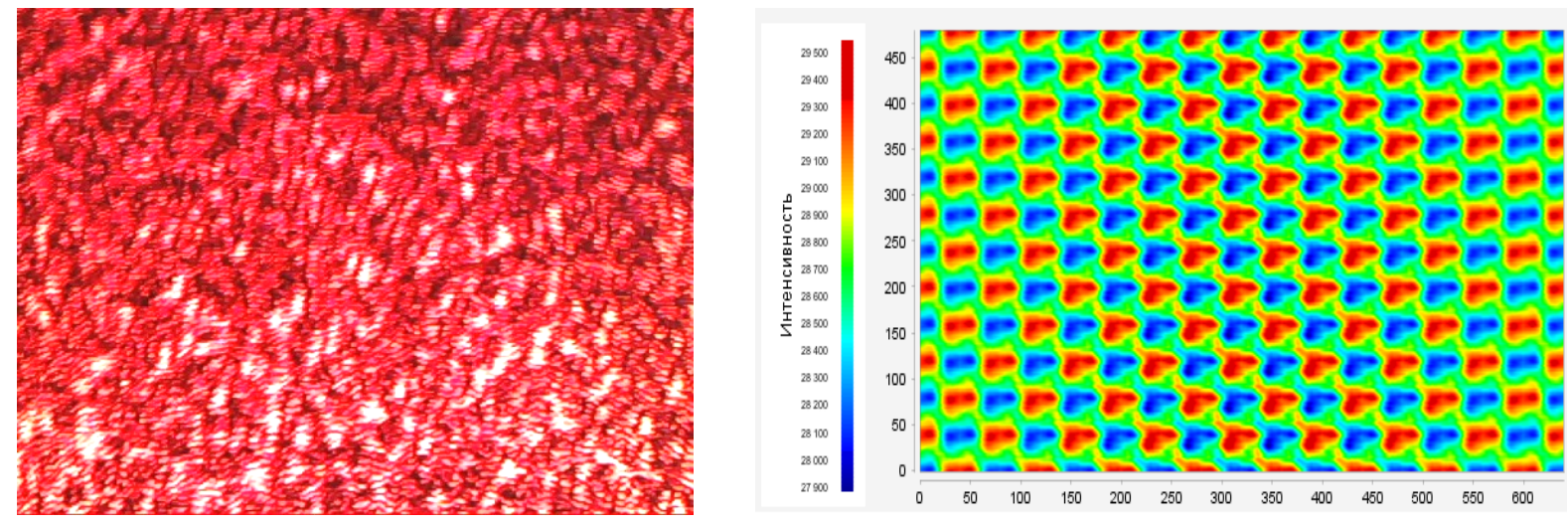

a
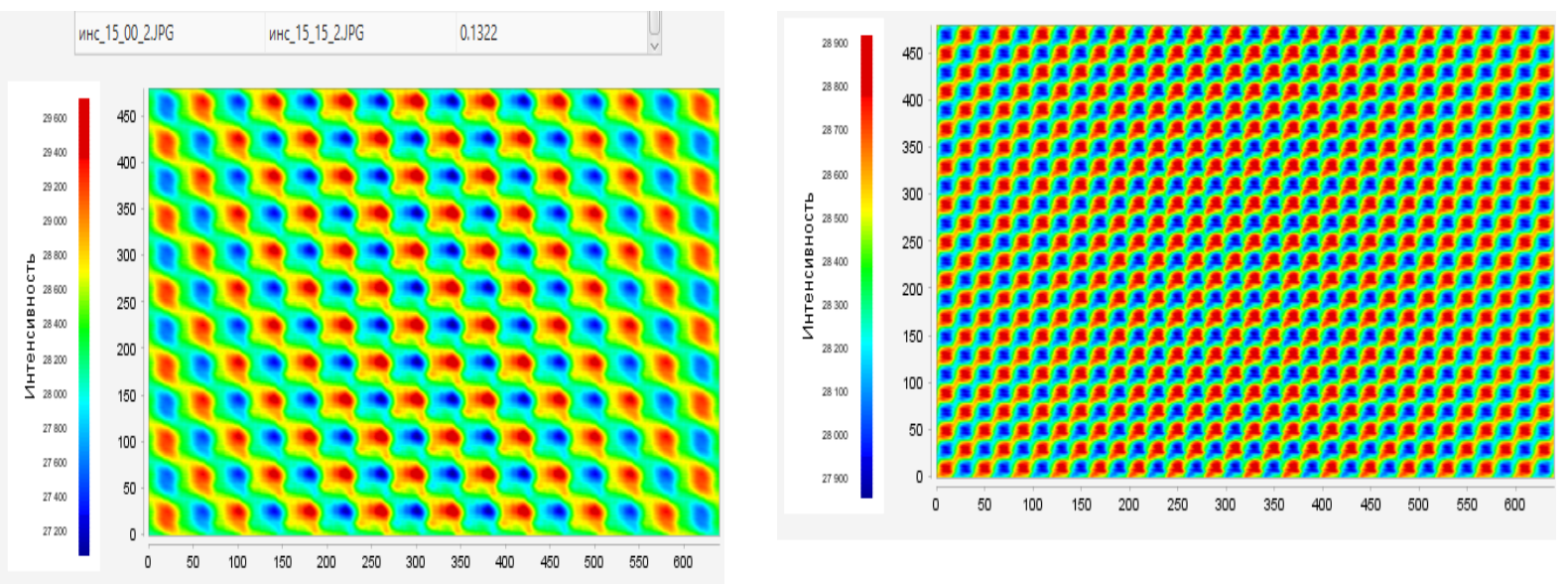

C

Fig. 2 A characteristic type of speckle pattern from the control sample (a), the correlation fields of the reference chessboard $(40 \times 40$ pixels) and the control speckle pattern (b), and the speckle pattern obtained after 15 minutes of laser exposure (c), the correlation field of the chessboard $20 \times 20$ pixels and the speckle pattern obtained after 15 minutes of laser exposure (d). Counts in pixels are situated on the vertical and horizontal axis of the correlation field, theamplitude of the correlation coefficient at each point of the field is indicated by color.

state changes of protein molecules in the solution. Before laser exposure, organic macromolecules in solution had an aqueous environment and formed clusters of different sizes and configurations: from very large to very small. During solution drying macromolecular clusters of large sizes (which as a whole had a lower rate of chaotic motion) were fixed on the substrate the first and they played the role of crystallization centers for the crystal growth in the film. Then smaller associates were deposited and, at the end, the smallest, that formed peaks due to the fact that their small mass allowed to stay on the top of the resulting peak.

After laser exposure the solution was homogenized: large clusters were divided into smaller ones, and the smallest clusters merged, became larger. Thus, the degree of cluster size variation decreased. During crystallization from a solution homogenized after laser exposure, the associates were placed in a smoother layer, since the share of minimum size clusters forming peaks decreased, and the number of peaks decreased too.

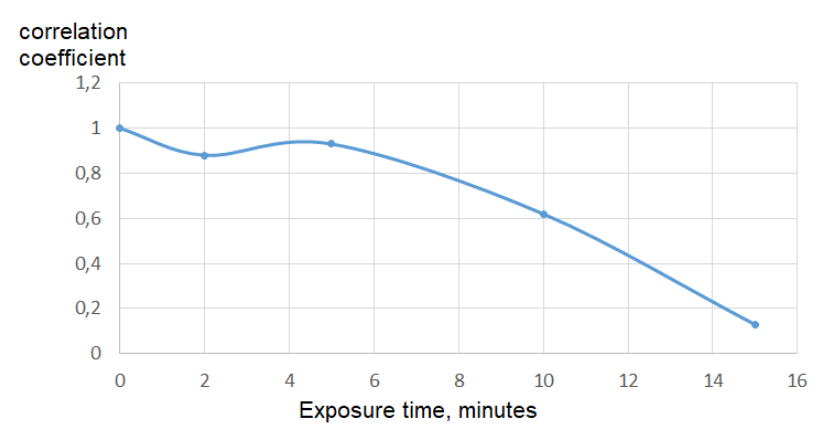

Fig. 3 The dependence correlation coefficient of the reference chessboard and speckle-patterns obtained at different time points.

Characteristic type of speckle pattern from the control sample, correlation fields between the reference chessboard $(40 \times 40$ pixels $)$ and the control speckle 
pattern and the speckle pattern obtained after 15 minutes of laser exposure are presented in Fig. 2. The correlation field of the speckle pattern obtained before irradiation had a clearly defined peak, while the correlation field of the speckle pattern obtained after 15 minutes of exposure had a peak with smaller amplitude and more blurred shape, which led to the characteristic maxima chains formation. It means that the characteristic speckle size did not match the square size on the "chessboard". For comparison, the correlation field of the speckle pattern obtained after 15 minutes of irradiation and a chessboard with a square size of $20 \times 20$ pixels are presented in Fig. 2(d). This field shows a set of clearly defined peaks, which indicates the equality of the chessboard squares $(20 \times 20$ pixels $)$ and speckle size. Thus, it can be argued that the characteristic size of the speckle varies from 40 to 20 pixels (or from 15 to 30 microns), which corresponds to the inhomogeneity size increasing by 2 times in the solution. In addition, the obtained result can be interpreted as the cluster size spread decreasing, with an increasing of the large clusters contribution to the speckle pattern formation.

There is correlation coefficient dependence of the reference checkerboard $(40 \times 40$ pixels $)$ and the speckle patterns obtained at different time points in Fig. 3. A decreasing of the correlation coefficient between the speckle pattern and the reference chessboard also indicates a changing of the characteristic speckle size. While the speckle size remained approximately equal to the square size of the "chessboard", the correlation coefficient had high values (0.8-1). After 5 minutes of laser exposure the microstructure of the insulin solution was modified, that caused the characteristic speckle size decreasing and this size became not equal to the chessboard square size and it led to a sharp reducing of the correlation coefficient.

\section{Conclusion}

It was shown experimentally that the insulin solution structure changed under action of $655 \mathrm{~nm}$ laser radiation. Analysis of insulin crystallograms with the help of probe microscope revealed a significant increase of surface smoothness due to irradiation. We supposed that laser exposure of the insulin solution caused such conformational changes which led to greater cluster size homogeneity in the solution. At the same time, the contribution of large clusters to the formation of speckle pattern increased, that led to a twofold reduction in the characteristic speckle size: from 40 to 20 pixels (which corresponded to a change in cluster size from 15 to $30 \mu \mathrm{m})$.

\section{Disclosures}

All authors declare that there is no conflict of interests in this paper.

\section{References}

1. A. V. Kartelishev, A. G. Rumyancev, A. R. Evstigneev, A. V. Geiniz, and S. V. Usova (Eds.), Lazernaya terapiya i profilaktika, Prakticheskaya meditsina, Moscow, Russia (2012) [in Russian]. ISBN 978-5-98811-219-8.

2. A. M. Enejder, T. W. Koo, J. Oh, M. Hunter, S. Sasic, M. S. Feld, and G. L. Horowitz, "Blood analysis by Raman spectroscopy", Optics Letters 27(22), 2004-2006 (2002).

3. A. Rygula, K. Majzner, K. M. Marzec, A. Kaczor, M. Pilarczyk, and M. Baranska, "Raman spectroscopy of proteins: a review", Journal of Raman Spectroscopy 44(8), 1061-1076 (2013).

4. G. A. Zalesskaya, E. G. Sambor, "Interaction of low-intensity laser radiation with blood and its components", Journal of Applied Spectroscopy 72(2), 230-235 (2005) [in Russian].

5. A. N. Malov, A. Y. Seteikin, A. V. Neupokoeva, E. S. Musatova, I. E. Golub, L. V. Sorokina, V. S. Fetschenko, and A. A. Vaichas, "The laser radiation action on the biological objects", Optik 124(23), 6034-6041 (2013).

6. R. A. Paringer, A. V. Kupriyanov, and N. Y. Ilyasova, "Dendritic crystallogram images classification", Journal of Biomedical Photonics \& Engineering 1(2), 135-138 (2015).

7. A. N. Malov, A. V. Neupokoeva, "A «chessboard» method for the speckle images analysis", Holography. Science and practice. Collection of works of the 10th International Conference "GoloExpo 2013", OOO «MNGS», Moscow, 257-264 (2013) [in Russian].

8. M. A. Vilensky, D. N. Agafonov, D. A. Zimnyakov, V. V. Tuchin, and R. A. Zdrazhevsky, "Speckle-correlation analysis of microcapillary blood flow in the nail bed," Quantum Electronics 41(4), 324-328 (2011).

9. S. V. Moskvin, Osnovy lazernoy terapii. Seriya "Effektivnaya lazernaya terapiya," Triada, Tver (2016) [in Russian].

10. A. N. Malov, A. V. Neupokoyeva, and L. A. Kokorina, "Laser modification of liquid nutrient medium and antibiotic solutions with the assessment by the bacteria growth dynamics," The Journal of Biomedical Photonics \& Engineering 3(2), 020303 (2017).

11. Y. Y. Tarasevich, "The models and mechanisms of the dehydration self-organization of biological fluids," PhysicsUspekhi 47(7), 717-728 (2004).

12. E. G. Rapis, "A change in the physical state of a nonequilibrium blood plasma protein film in patients with carcinoma," Technical Physics 47(4), 510-512 (2002). 
13. A. I. Andryushkin, S. P. Sapozhnikov, and A. V. Karpunina, "Crystallography of biological fluids (a review)," Vestnik Chuvashskogo Universiteta 3, 355-359 (2013) [in Russian].

14. P. V. Pavlov, A. N. Malov, A. V. Neupokoeva, and F. N. Popov, "A testing method for machine details state by means of the speckle image parameters analysis”, Proceedings of SPIE 10176, 101760Z (2016). 